[in press at Cognition; accepted April 1, 2015]

\title{
Space and Time in the Sighted and Blind
}

Roberto Bottini ${ }^{1}$, Davide Crepaldi ${ }^{2,3}$, Daniel Casasanto ${ }^{4}$, Virgine Crollen ${ }^{5,6} \&$ Olivier Collignon ${ }^{1}$

Word count:

Abstract: 220

Main text: 3106

${ }^{1}$ Centre for Mind/Brain Science, University of Trento, Italy

${ }^{2}$ MoMo Lab, Department of Psychology, University of Milan-Bicocca, Italy

${ }^{3}$ Milan Center for Neuroscience (NeuroMi)

${ }^{4}$ Department of Psychology, University of Chicago, IL, USA

${ }^{5}$ Institut de Recherche en Sciences Psychologiques (IPSY), Centre de Neuroscience Système et Cognition (NeuroCS), Université Catholique de Louvain, Belgium

${ }^{6}$ Centre de Recherche en Neuropsychologie et Cognition (CERNEC), Université de Montréal, Canada

Corresponding Author:

Roberto Bottini, Ph.D.

CIMeC - Center for Mind/Brain Sciences

University of Trento

Via delle Regole 101

38123, Mattarello (TN)

Italy

Phone: +390461283010

bottini.r@gmail.com 


\section{Abstract}

Across many cultures people conceptualize time as extending along a horizontal Mental Time Line (MTL). This spatial mapping of time has been shown to depend on experience with written text, and may also depend on other graphic conventions such as graphs and calendars. All of this information is typically acquired visually, suggesting that visual experience may play an important role in the development of the MTL. Do blind people develop a MTL? If so, how does it compare with the MTL in sighted? In this study we tested early blind, late blind and sighted participants in a space-time congruity task. Participants had to classify temporal words by pressing a right and a left key, either with crossed or uncrossed hands. We found that the MTL develops in the absence of vision, and that it is based on the same external frame of reference in sighted and blind people. Reading braille may provide the same experiential link between space and time in the manual modality as reading printed text provides in the visual modality. These results showing a similar MTL in sighted and blind participants contrast with previous results showing that the Mental Number Line (MNL) depends on different spatial coordinates in the sighted and the blind, and suggest that spatial representations of time and number may have different experiential bases.

Keywords: Time; Space; Blindness; Frame of reference; Orthography. 


\section{Introduction}

Time and space are tightly intertwined in the human mind. For instance, temporal order is often represented in the mind by mean of a Mental Time Line (MTL) in which earlier and later events are mapped onto the left and right side of space, respectively. In Western cultures, people are faster to categorize earlier events by pressing a left key and later events pressing a right key compared to vice-versa (Casasanto \& Bottini, 2014; Fuhrman \& Boroditsky, 2010; Santiago, Lupiáñez, Pérez, \& Funes, 2007; Urlich \& Maienborn, 2010; Weger \& Pratt, 2008). Accordingly, induced rightward or leftward biases of visual-spatial attention influence temporal judgments (Frassinetti, Magnani, \& Oliveri, 2009; Vicario, Pavone, Martino \& Fuggetta, 2011; Vicario, Caltagirone, \& Oliveri, 2007), patients with left spatial neglect also neglect the "left side" of time (Saj et al., 2013), and people spontaneously gesture according to the MTL when talking about temporal relationships (Casasanto \& Jasmin, 2012).

The experience of reading and writing seems to play a role in establishing the direction and orientation of the MTL (Casasanto \& Bottini, 2014; Fuhrman \& Boroditsky, 2010; Ouellet, Santiago, Israeli \& Gabay, 2010; Tversky, Kugelmass \& Winter, 1991). Events unfold rightward along the MTL in people who habitually read from left to right, and leftward in people who read from right to left (e.g. Israeli Hebrew-speakers; Fuhrman \& Boroditsky, 2010; Ouellet et al. 2010). Beyond showing a correlation between reading direction and the MTL, a training experiment demonstrated a causal role for reading experience in determining which direction time flows in people's minds. Exposing people who usually read from left to right to mirrorreversed orthography reversed the direction of their MTLs (Casasanto \& Bottini, 2014).

Why does reading experience influence the MTL? As people read, they move their eyes and attention 'through' both space and time. For each line of text in English, readers begin on the 
left of the page at an earlier time, and arrive on the right of the page at a later time. This experience is apparently sufficient to cause earlier time points to become implicitly associated with one side of space and later time points with the other (Casasanto \& Bottini, 2014). Other cultural conventions that tend to covary with orthography may also contribute to this space-time association; these may include graphic representation of time in calendars and graphs, temporal sequences in comic strips, and spontaneous gestures toward the past or the future. Crucially, all of this information is acquired visually, suggesting that visual input plays an important role in shaping the MTL (Bonato, Zorzi \& Umiltà, 2012), at least in the people who have been tested to date. Yet, it is unclear whether visual experience per se is an "active ingredient" in the development of the MTL. Do people who have never experienced functional vision develop a MTL? If so, how does it compare with the MTL in sighted people?

One possibility is that the experience of moving systematically through both time and space in the act of reading is sufficient to determine the spatial characteristics of the MTL, regardless of the sensory modality in which reading occurs. Although blind people have limited access to some graphic representations of time, and cannot see co-speech gestures, many blind people have reading experience that is similar to visual reading in the aspects that are believed to be relevant for establishing a MTL. In reading braille text, which is conventionally written from left to right, the hand (or hands) moves rightward across the page following the direction of the orthography. Reading braille, therefore, may provide the same experiential link between space and time as reading printed text. If the development of the MTL depends on orthographic experience across modalities, then sighted and blind individuals should have similarly organized MTLs. 
Alternatively, vision, or lack thereof, may determine the spatial characteristics of the MTL. Specifically, the way blind people experience spatial relationships sensorially may cause their temporal concepts to be constructed differently than their sighted counterparts', regardless of reading experience. Several experiments have shown that the mental organization of nonvisual spatial frames of reference (FoR) in early blind (who lost their sight before age $\sim 3$ ) is qualitatively different compared to sighted people (Crollen \& Collignon, 2012). Studies of tactile stimulus localization (Röder, Rösler \& Spence, 2004) and multisensory control of action (Collignon, Charbonneau, Lassonde \& Lepore, 2009; Röder, Kusmierek, Spence \& Schicke, 2007) show that whereas sighted people tend to rely on an external spatial FoR (i.e., locations are represented within a framework external to the body), the early blind preferentially use an anatomical FoR (i.e., locations are represented with respect to the position of one's body and the position of one's limbs), to represent spatial relationships.

Does the difference between blind and sighted people's default FoR extend to the use of space for representing abstract concepts, in non-spatial domains? A study in the domain of numbers suggests that it does. Like temporal relationships, numerical relationships are also represented spatially by means of a horizontal Mental Number Line (MNL) where lower numbers are associated with the left side of space and higher numbers with the right side (Dehaene, Bossini \& Giraux, 1993). Although a space-number mapping can also be observed in the early blind (Castronovo \& Seron, 2007), they use a different FoR to represent numbers compared to sighted or late blind (who lost their sight after age 3 ; Crollen, et al. 2013). In a space-number congruity task, participants pressed buttons on the left and right side of space to judge number magnitude. Results showed that for the sighted and late blind participants, lower numbers were implicitly associated with the left side of space and higher numbers with the right 
side. This was true no matter whether they pressed the buttons using a typical uncrossed hand posture (left hand on the left button and right hand on the right button) or with their hands crossed (left hand on the right button and right hand on the left button), indicating that they were mapping number onto an external FoR. By contrast, in early blind participants the space-number congruity effect reversed between the uncrossed and crossed-hands conditions. Lower numbers were implicitly associated with the left hand, and higher numbers with the right hand, indicating that they mapped number onto an anatomical, hand-based FoR. Do early blind also use a handbased FoR to represent time?

Although numerous studies have documented the MTL in sighted people, to date no study has tested whether blind people also organize time according to an implicit MTL. Furthermore, no study has evaluated the spatial FoR used for temporal order, in any population. ${ }^{1}$ In this experiment early blind, late blind and sighted individuals performed a temporal classification task by pressing two keys positioned on the left and the right side of space. In one condition their hands were uncrossed, and in the other condition they were crossed. We hypothesized that, since the direction of the MTL appears to be determined by reading experience in sighted participants, their MTL should be represented in external spatial coordinates: As people read Italian, they start on the left of each line of text and end on the right, regardless of the positions of their hands. Thus, sighted participants should show a similar spacetime congruity effect no matter whether their hands are uncrossed or crossed: past times should be implicitly associated with the left side of space, and future times with the right side.

\footnotetext{
${ }^{1}$ One previous study showed that space-time congruity effects were unchanged in the uncrossed vs. crossed hands condition (Vallesi, et al, 2008). However, this earlier study concerned a different aspect of time: duration (i.e. temporal magnitude), rather than temporal order.
} 
For blind participants, we considered three possibilities. First, if their MTLs are constructed similarly to the (expected) MTLs in sighted participants, on the basis of their experience reading braille, then they should use an external FoR, and show a similar space-time congruity effect across postures. Alternatively, early blindness may lead to the default use of an anatomical FoR to map abstract concepts onto space (as in the case of numbers). If so, for (early) blind participants the past should be implicitly associated with the left hand, and the future with the right hand. Finally, blind participants could show no space-time congruity effect, at all. This outcome would suggest that vision is necessary to establish a left-right spatial representation of temporal order.

\section{Method}

\subsection{Participants}

One group of 16 sighted and two groups of blind participants (17 early and 16 late blinds) took part in the study (see Table 1 for details). The three groups did not statistically differ in terms of age (all p-values >.05). Participants in both blind groups were totally blind or had only rudimentary sensitivity for brightness differences. In all cases, blindness was attributed to peripheral deficits with no additional neurological problems. The experiment was approved by the Research Ethics Boards of the University of Milan-Bicocca. All participants were Italian native speakers and were blindfolded when performing the tasks. 
Table 1. Characteristics of the blind participants

\begin{tabular}{|c|c|c|c|c|c|}
\hline Participants & Gender & Age & Handedness (EHI) & Onset total blindness & Cause of blindness \\
\hline EB1 & M & 49 & 0.42 & 0 & Congenital retinitis pigmentosa \\
\hline EB2 & $\mathrm{F}$ & 51 & 0.54 & 0 & Rubella of the mother during pregnancy \\
\hline EB3 & $\mathrm{F}$ & 33 & 1 & 0 & Bilateral congenital microphthalmia \\
\hline EB4 & $\mathrm{F}$ & 50 & 0.9 & 1 & Retinoblastoma \\
\hline EB5 & $\mathrm{F}$ & 76 & 0.83 & 2 & Sepsis \\
\hline EB6 & M & 51 & 0.81 & 3 & Primary congenital glaucoma \\
\hline EB7 & M & 25 & 1 & 0 & Retinoplasia \\
\hline EB8 & M & 34 & 0.73 & 0 & Retinopathy of prematurity \\
\hline EB9 & M & 29 & 0.74 & 0 & Optic nerve hypoplasia \\
\hline EB10 & M & 45 & 0.91 & 0 & Congenital retinitis pigmentosa \\
\hline EB11 & M & 52 & 0.82 & 0 & Primary congenital glaucoma \\
\hline EB12 & M & 33 & 0.48 & 0 & Retinopathy of prematurity \\
\hline EB13 & M & 27 & 0.58 & 0 & Norrie syndrome \\
\hline EB14 & M & 45 & 0.17 & 0 & Retinopathy of prematurity \\
\hline EB15 & $\mathrm{F}$ & 26 & 0.91 & 2 & Retinoblastoma \\
\hline EB16 & $\mathrm{F}$ & 44 & 0.48 & 2 & Retinoblastoma \\
\hline EB17 & $\mathrm{F}$ & 22 & 0.39 & 0 & Congenital retinitis pigmentosa \\
\hline LB1 & M & 74 & 0.90 & 33 & Retinitis Pigmentosa \\
\hline LB2 & $\mathrm{F}$ & 27 & 0.76 & 19 & Rheumatoid Arthritis \\
\hline LB3 & $\mathrm{F}$ & 68 & 0.90 & 30 & Retinits Pigmentosa \\
\hline LB4 & M & 56 & 0.91 & 11 & Glaucoma \\
\hline LB5 & $\mathrm{F}$ & 33 & 0.53 & 20 & Retinitis Pigmentosa \\
\hline LB6 & M & 45 & 1 & 22 & Retinitis Pigmentosa \\
\hline LB7 & $\mathrm{F}$ & 48 & 1 & 30 & Retinitis Pigmentosa \\
\hline LB8 & $\mathrm{F}$ & 26 & 0.24 & 7 & Brain cancer \\
\hline LB9 & $\mathrm{F}$ & 60 & 0.70 & 20 & Glaucoma \\
\hline LB10 & $\mathrm{F}$ & 58 & 0.75 & 18 & Retinopathy of prematurity \\
\hline LB11 & M & 59 & 0.91 & 10 & Retinitis pigmentosa \\
\hline LB12 & M & 50 & 0.56 & 12 & Uveitis \\
\hline LB13 & M & 35 & 0.36 & 3 & Retinopathy of prematurity \\
\hline LB14 & $\mathrm{F}$ & 54 & 0.68 & 21 & Glaucoma \\
\hline LB15 & M & 49 & 1 & 20 & Glaucoma \\
\hline LB16 & $\mathrm{M}$ & 55 & 1 & 5 & Glaucoma \\
\hline
\end{tabular}

$\mathrm{M}=$ male; $\mathrm{F}=$ female; EHI: $<-0.5=$ left-handed; $>0.5=$ right-handed; $-0.5><0.5=$ ambidextrous

\subsection{Materials and Procedure}

Participants were asked to classify orally presented Italian words as "referring to the past" or "referring to the future". Past-related words were: "passato" (past), "prima" (earlier), "ieri" 
(yesterday), "scorso" (previous). Future-related words were: "futuro" (future), "dopo" (later), "domani" (tomorrow), "prossimo" (next). Stimuli lasted $600 \mathrm{msec}$, had identical auditory properties $(44,100 \mathrm{~Hz}, 16$ bits, stereo), and were played through loudspeakers placed in front of the participant. Participants were instructed to respond as quickly and accurately as possible by pressing one of two response keys placed $30 \mathrm{~cm}$ in front of each participant's body and $20 \mathrm{~cm}$ away from the body midline in the left and right hemi-spaces. The task comprised two response assignments. In the first condition, the "past" response was assigned to the left response key, while the "future" response was assigned to the right response key (response code congruent with the MTL). In the second condition, the reverse assignment was used: the "future" response to the left key and the "past" response to the right key (response code incongruent with the MTL). Moreover, participants were asked to perform the task either with their hands in a uncrossed posture or with their arms crossed over the body midline so that the left hand was on the right response key and the right hand was on the left response key (see Fig. 1). Each participant completed 4 blocks of trials [response mode (2) X posture (2)]. The order of response mode and posture conditions was counterbalanced across participants. During testing, subjects sat in a silent room with the head restrained by a chin rest. Stimuli were delivered and reaction times were recorded using MatLab Psychtoolbox (Brainard, 1997; Pelli, 1997). Each word was presented 16 times in each condition, giving a total of 512 stimuli randomly presented in 4 experimental blocks. The inter-stimuli interval ranged from 1500 to $2500 \mathrm{msec}$. 


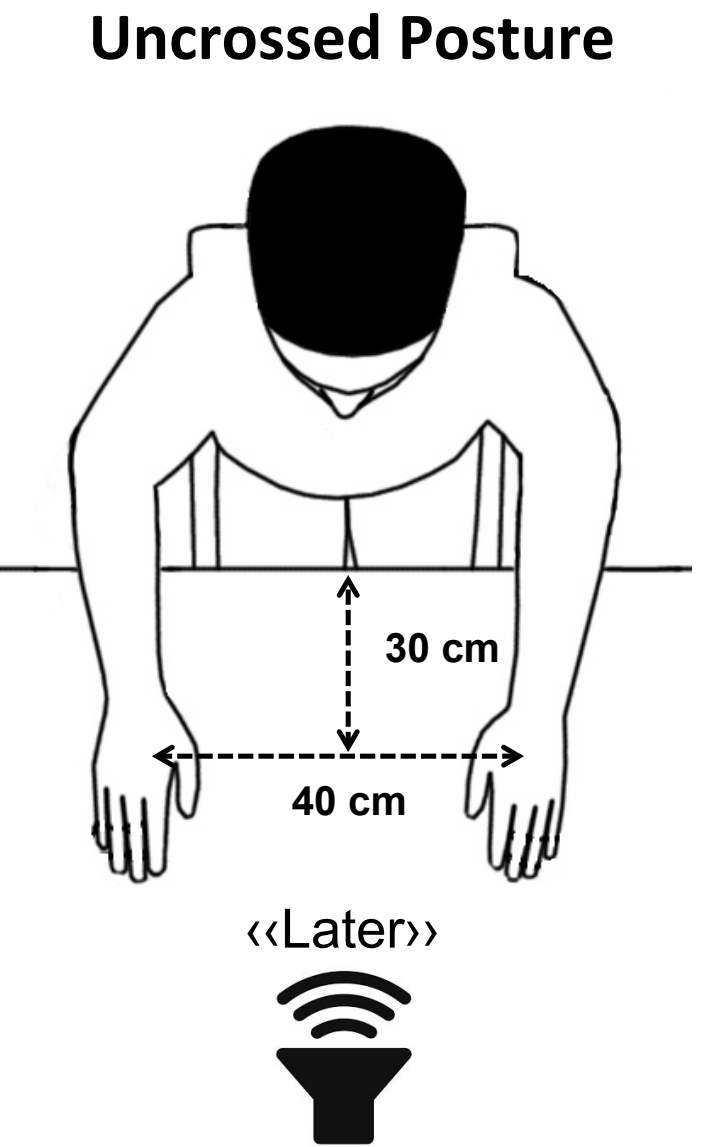

\section{Crossed Posture}

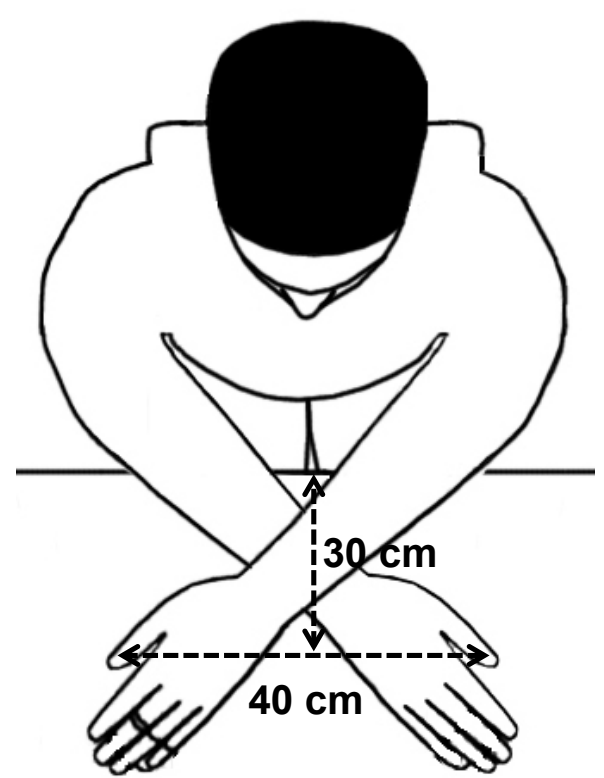

«Later»

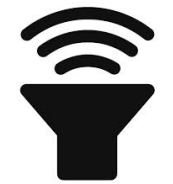

Figure 1. Hand postures participants used in the uncrossed and crossed-hands versions of the space-time congruity task.

\section{Results}

Incorrect responses $(\sim 1 \%)$ were excluded from the analysis. For each subject, RTs that exceeded for $+/-2$ SD from the mean were also excluded ( $\sim 3 \%$ of the correct trials). The logarithms of RTs were analyzed using linear mixed-effects regressions (LME). Random intercepts were included for subjects and items in all the regression models, so that any subjectspecific and item-specific variability was taken into account separately and did not contribute to the overall error. 
Sighted participants showed a significant main effect of congruity (Wald $\chi^{2}(1)=30.86 p<$ .001 ), indicating that participant was faster in the condition congruent with the MTL (past-left, future-right) than in the incongruent condition (Fig. 2). There was also a main effect of posture (Wald $\chi^{2}(1)=75.68 \mathrm{p}<.001$ ), indicating faster RTs in the uncrossed compared to the crossed posture. There was no Congruity by Posture interaction (Wald $\left.\chi^{2}(1)=1.07, p=.30\right)$, indicating that posture (uncrossed, crossed) did not modulate the congruity effect. Pairwise comparisons showed that the effect of Congruity was significant both in the uncrossed $\left(20 \mathrm{~ms}\right.$; Wald $\chi^{2}(1)=$ $25.74 \mathrm{p}<.001)$ and in the crossed posture $\left(15 \mathrm{~ms} ;\right.$ Wald $\left.\chi^{2}(1)=11.02 \mathrm{p}<.001\right)$.

Late blind showed a significant congruity effect overall (Wald $\left.\chi^{2}(1)=80.11 \mathrm{p}<.001\right)$, no effect of Posture (Wald $\chi^{2}(1)=1.66 \mathrm{p}=.19$ ) and no significant Posture by Congruity interaction (Wald $\left.\chi^{2}(1)=1.20 \mathrm{p}=.27\right)$. The effect of Congruity was significant both in the uncrossed (41ms; Wald $\left.\chi^{2}(1)=54.87 \mathrm{p}<.001\right)$ and in the crossed posture $\left(36 \mathrm{~ms}\right.$; Wald $\left.\chi^{2}(1)=31.72 \mathrm{p}<.001\right)$. Early blind showed a significant congruity effect overall (Wald $\chi^{2}(1)=51.81 \mathrm{p}<.001$ ) a significant main effect of posture (Wald $\chi^{2}(1)=33.93 \mathrm{p}<.001$ ) but no congruity by posture interaction (Wald $\chi^{2}(1)=2.53, \mathrm{p}=.11$ ). The effect of Congruity was significant both in the uncrossed $\left(26 \mathrm{~ms}\right.$; Wald $\left.\chi^{2}(1)=18.85 \mathrm{p}<.001\right)$ and in the crossed posture $\left(29 \mathrm{~ms}\right.$; Wald $\chi^{2}(1)=$ $36.96, \mathrm{p}<.001)$.

The 3-way interaction Congruity by Posture by Group did not reach significance (Wald $\left.\chi^{2}(2)=4.84, p=.09\right)$. 


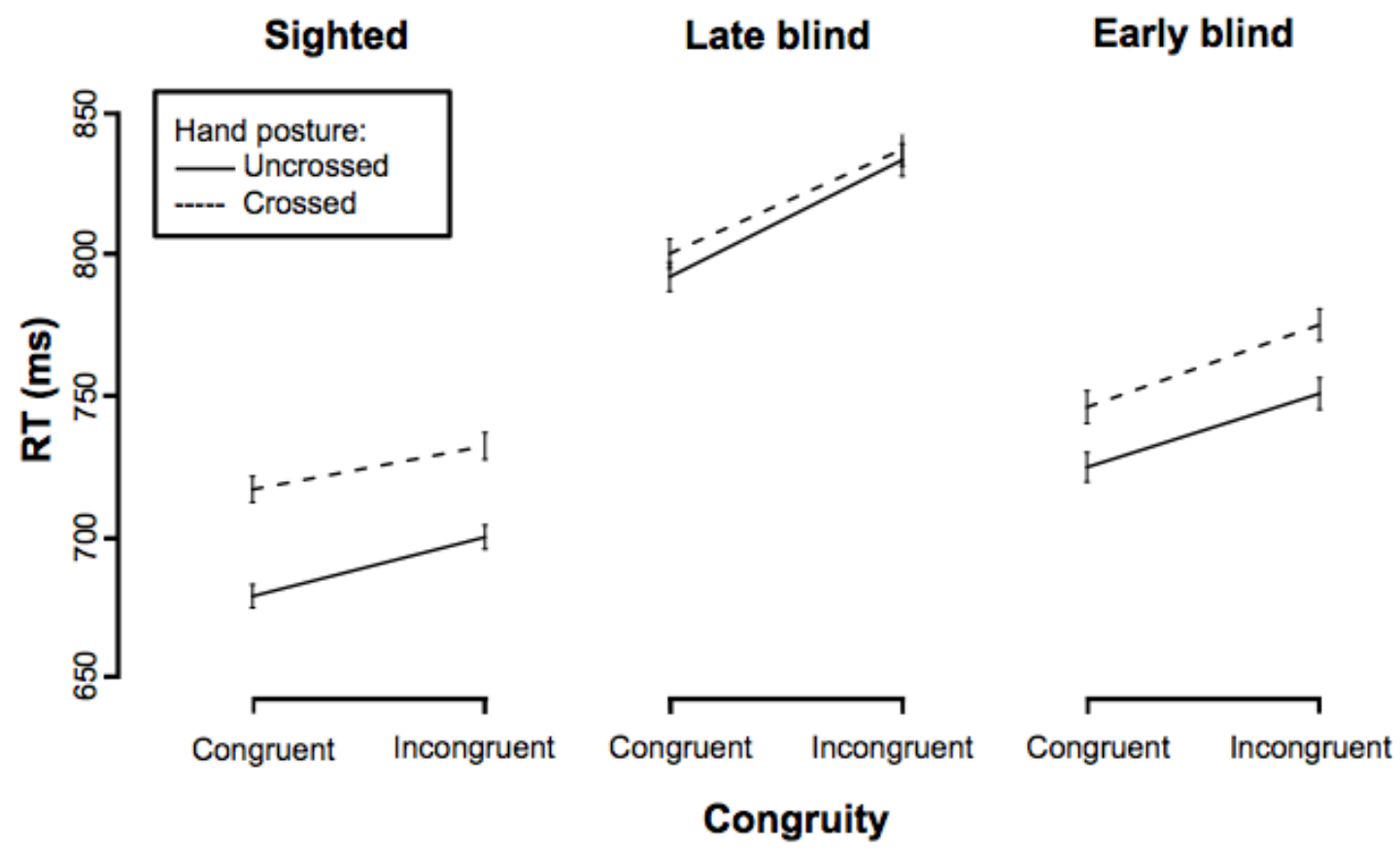

Figure 2. RTs of sighted, late blind and early blind participants in the space-time congruity task. The congruity effect (faster RTs when the position of the response keys is congruent with a rigthward MTL) does not vary across posture conditions. Error bars indicate standard errors of the mean.

\section{Discussion}

This study provided the first test of a Mental Time Line in blind people, and the first test of the spatial coordinate system used for the MTL in both sighted and blind. Our results show that the MTL is mapped onto external spatial coordinates in sighted individuals: Past events were associated with the left side of space and future events with the right side, regardless of the participants' hand posture. Blind participants showed a similar pattern of results, establishing that vision is not necessary for the development of an MTL: Even early blind individuals showed a rightward-directed MTL, much like their sighted counterparts. Finally, we showed that early blindness does not necessarily lead to the default use of an anatomical FoR to map abstract 
concepts onto space. Unlike the mental number line (Crollen et al., 2013), the mental timeline is represented in external spatial coordinates in both the sighted and the blind.

The use of an external FoR for representing temporal order is consistent with the hypothesis that the direction of the MTL depends on orthographic experience (Casasanto \& Bottini, 2014; Fuhrman \& Boroditsky, 2010; Ouellet et al., 2010). Reading text from left to right, whether with the eyes or the hands, leads to a similar horizontal representation of temporal order in which earlier times are located to the left of later times, independent of the position of one's hands. The existence of a causal link between the MTL and reading experience (Casasanto \& Bottini, 2014) may explain why early blind people represent the MTL in external spatial coordinates, and not anatomical spatial coordinates as for the MNL. That is, the general tendency to use an anatomical FoR for representing nonvisual spatial coordinates in early blind might be overcome when external spatial coordinates are highly salient, as in reading. Our data are in accord with data from perceptual-motor tasks in which early blind participants have been found, under some circumstances, to adopt an external FoR like their sighted counterparts. For instance, both sighted and blind people rely onto an external FoR for coordinating bimanual movements such as finger tapping and finger oscillation (Heed \& Röder, 2014). Additionally, it has been shown that also sighted people tend to rely on different FoRs to estimate the location of a tactile stimulus depending on the salience of a particular frame of reference (external or anatomical) in a secondary task (Badde, Röder \& Heed, 2014). In the case of action control and spatial localization of stimuli, different frames of reference appear to be deployed flexibly on the basis of task requirements; the same may be true when spatial schemas are used to represent abstract concepts. 
Parallels are often drawn between the MTL and the MNL (Bonato et al. 2012; Walsh, 2003). Yet, taken together with the results of an analogously constructed study of the MNL by our research group (Crollen et al. 2013), the present results reveal a fundamental distinction between spatial mappings of time and number. Crollen and colleagues found that the MNL depends on different spatial coordinates in the sighted and the blind, whereas we find that the MTL depends on the same spatial coordinates, in all groups. This result is consistent with the claim that the MTL and MNL may have different experiential origins (Pitt \& Casasanto, 2014). That is, compared to the MTL, the MNL may be less dependent on experience reading text. Whereas the direction of the MTL has been shown to reverse after brief exposure to mirror-reversed text (Casasanto \& Bottini, 2014), mirror reading had no effect on the direction of the MNL (Dehaene et al, 1993). Furthermore, reading direction is not necessarily predictive of the direction of the MNL: Israeli Hebrew speakers, who read text from right to left, do not show a reversed MNL (Shaki, Fischer, \& Petrusic, 2009). The MNL appears to depend on other forms of culturespecific experience, such reading numbers that increase from left to right or vice versa (Fischer, Mills, \& Shaki, 2010), and finger counting habits (Fischer, 2008; Pitt \& Casasanto, 2014). In the early blind, as in the sighted, the MTL may be more dependent on the experience of reading text than the MNL is. This difference could explain why time appears to be mapped by default onto an external coordinate system in the blind but number does not.

\section{Conclusions}

Blind participants appear to conceptualize time using a horizontal mental time line, much like their sighted counterparts. Sighted and blind participants organized temporal succession along a MTL based on an external spatial FoR. These findings contrast with those of an analogous study 
on the spatial mapping of number (Crollen et al. 2013) showing that the mental number line depends on different spatial coordinates in the sighted (external FoR) and the blind (anatomical FoR). It has remained an open question whether the spatial mappings of number and time are truly distinct, or whether they are manifestations of a single propensity to represent continuous information from abstract domains along a spatial continuum (Bonato et al. 2012; Walsh, 2003). If the MTL and MNL were underlyingly the same, then they should be based on the same spatial FoR, and changes in people's experiences (e.g., due to sensory deprivation) should have similar effects on the spatial FoRs used for time and for number. This does not appear to be the case. By extending the study of space-time and space-number congruity effects to the blind, we find that visual experience has different effects on the spatial coordinate system used to represents the MTL and the MNL, therefore suggesting they are not necessarily relying on the same mechanisms. These findings underscore the need to distinguish spatial mappings of time and number in theorizing about the spatial basis of abstract concepts.

\section{Acknowledgments}

We wish to thank blind volunteers, the Italian Union for Blind and Visually Impaired and the Institute for the Blind of Milano for their collaboration. We also thank Jessica Facoetti for her help in collecting the data. Research was supported in part by a European Research Council starting grant (MADVIS; ERC-StG 337573) attributed to O. Collignon; a FIRB-Futuro in Ricerca granted by the Italian Ministry of Education, University and Research (RBFR085K98) to D. Crepaldi; a James S. McDonnell Foundation Scholar Award (\#220020236) to D. Casasanto. V. Crollen is a post-doctoral researcher supported by the National Fund for Scientific Research (Belgium). 


\section{References}

Badde, S., Röder, B., Heed, T. (2014). Flexibly weighted integration of tactile reference frames. Neuropsychologia. doi:10.1016/j.neuropsychologia.2014.10.001

Bonato, M., Zorzi, M., and Umiltà, C. (2012). When time is space: evidence for a mental time line. Neuroscience and Biobehavioral Review, 36, 2257-2273.

Brainard, D. H. (1997) The Psychophysics Toolbox, Spatial Vision , 10:443-446.

Casasanto, D. \& Bottini, R. (2014). Mirror-reading can reverse the flow of time. Journal of Experimental Psychology: General. 143(2), 473-9.

Casasanto, D. \& Jasmin, K. (2012). The Hands of Time: Temporal gestures in English speakers. Cognitive Linguistics, 23(4), 643 - 674.

Castronovo, J., \& Seron, X. (2007). Semantic numerical representation in blind subjects: the role of vision in the spatial format of the mental number line. The Quarterly journal of experimental psychology, 60(1), 101-119.

Collignon, O., Charbonneau, G., Lassonde, M., \& Lepore, F. (2009). Early visual deprivation alters multisensory processing in peripersonal space. Neuropsychologia, 47(14), 32363243.

Crollen, V., \& Collignon, O. (2012). Embodied space in early blind individuals. Frontiers in Psychology, 3(August), 272.

Crollen, V., Dormal, G., Seron, X., Lepore, F., \& Collignon, O. (2013). Embodied numbers: The role of vision in the development of number-space interactions. Cortex, 49(1), 276-283.

Dehaene, S., Bossini, S., \& Giraux, P. (1993). The mental representation of parity and number magnitude. Journal of Experimental Psychology: General, 122(3), 371. 
Fischer, M. H. (2008). Finger counting habits modulate spatial-numerical associations. Cortex, 44(4), 386-92.

Fischer, M. H., Mills, R. A., \& Shaki, S. (2010). How to cook a SNARC: Number placement in text rapidly changes spatial-numerical associations. Brain and cognition, 72(3), 333-336.

Frassinetti, F., Magnani, B., \& Oliveri, M. (2009). Prismatic Lenses Shift Time Perception. Psychological Science, 20(8), 40127.

Fuhrman, O., \& Boroditsky, L. (2010). Cross-cultural differences in mental representations of time: Evidence from an implicit non-linguistic task. Cognitive Science, 34, 1430-1451.

Heed, T., \& Röder, B. (2014). Motor coordination uses external spatial coordinates independent of developmental vision. Cognition, 132(1), 1-15.

Ouellet, M., Santiago, J., Israeli, Z., \& Gabay, S. (2010). Is the future the right time? Experimental Psychology, 57, 308-314.

Pelli, D. G. (1997) The VideoToolbox software for visual psychophysics: Transforming numbers into movies, Spatial Vision, 10:437-442.

Pitt, B. \& Casasanto, D. (2014). Experiential Origins of the Mental Number Line. In P. Bello, M. Guarini, M. McShane, \& B. Scassellati (Eds.), Proceedings of the 36th Annual Conference of the Cognitive Science Society (pp. 1174-1179). Austin, TX: Cognitive Science Society.

Röder, B., Kusmierek, A., Spence, C., \& Schicke, T. (2007). Developmental vision determines the reference frame for the multisensory control of action. Proceedings of the National Academy of Sciences of the United States of America, 104(11), 4753-8. 
Röder, B., Rösler, F., \& Spence, C. (2004). Early Vision Impairs Tactile Perception in the Blind. Current Biology, 14(2), 121-124.

Saj, A., Fuhrman, O., Vuilleumier, P., \& Boroditsky, L. (2014). Patients with left spatial neglect also neglect the "left side" of time. Psychological science, 25(1), 207-214.

Santiago, J., Lupiãnez, J., Pérez, E., Funes, M.J., 2007. Time, also flies from left to right. Psychonomic Bulletin \& Review 14, 512-516.

Shaki, S., \& Gevers, W. (2011). Cultural characteristics dissociate magnitude and ordinal information processing. Journal of cross-cultural psychology, 42(4), 639-650.

Shaki, S., Fischer, M. H., \& Petrusic, W. M. (2009). Reading habits for both words and numbers contribute to the SNARC effect. Psychonomic Bulletin \& Review, 16(2), 328-331.

Tversky, B., Kugelmass, S., \& Winter, A. (1991). Cross-cultural and developmental trends in graphic productions. Cognitive Psychology, 23, 515-557.

Ulrich, R., \& Maienborn, C. (2010). Left-right coding of past and future in language: The mental timeline during sentence processing. Cognition, 117(2), 126-138.

Vallesi, A., Binns, M. A., \& Shallice, T. (2008). An effect of spatial-temporal association of response codes: Understanding the cognitive representations of time. Cognition, 107(2), $501-527$.

Vicario, C. M., Pavone, E. F., Martino, D., \& Fuggetta, G. (2011). Lateral head turning affects temporal memory. Perceptual and motor skills, 113(1), 3-10.

Vicario, C. M., Caltagirone, C., \& Oliveri, M. (2007). Optokinetic stimulation affects temporal estimation in healthy humans. Brain and Cognition, 64(1), 68-73. 
Walsh, V. (2003). A theory of magnitude: common cortical metrics of time, space and quantity. TiCS, 7(11),483-488.

Weger, U. W., \& Pratt, J. (2008). Time flies like an arrow: Space-time compatibility effects suggest the use of a mental timeline. Psychonomic Bulletin \& Review, 15(2), 426-430. 